\title{
Fibrinólisis en un paciente con trombosis de prótesis valvular
}

\section{Fibrinolysis in a patient suffering from thrombosis of valve prosthesis: Cases and literatare review}

\author{
Carol Salcedo ${ }^{1}$, Abner Lozano L. ${ }^{2}$, Jaime A. Salcedo ${ }^{3}$
}

Palabras clave: trombosis valvular, trombólisis, remplazo valvular.

Key words: valve thrombosis, thrombolysis, valve replacement.

\begin{abstract}
Resumen
La cardiopatía reumática y la fiebre reumática son las principales enfermedades que afectan las válvulas cardiacas y que progresan en reemplazo de estas, existen muchas variantes en el diseño de las prótesis valvulares y la durabilidad de estas. Sin embargo, la obstrucción de la válvula protésica sigue siendo una complicación con un riesgo alto de mortalidad; por lo cual se plantean diferentes estrategias terapéuticas y quirúrgicas que a pesar de la evidencia siguen siendo un tema controversial para el abordaje de un paciente como lo es la terapia de trombólisis versus cirugía. A continuación se presenta el caso clínico de un paciente de 15 años al que se le realiza el diagnostico de doble trombosis valvular quien posteriormente se lleva a fibrinólisis recibiendo estreptoquinasa sin complicación y manejándose posteriormente con terapia de anticoagulación ambulatoria.
\end{abstract}

\begin{abstract}
Rheumatic heart disease and rheumatic fever are the main diseases affecting the heart valves and progressing to replace them. There are many variants in the design of prosthetic valves and their durability. However, the prosthetic valve obstruction remains a complication with high mortality risk. For this reason, different therapeutic and surgical strategies, like thrombolysis therapy versus surgery, are proposed which despite evidence it remains a controversial issue to approach a patient. Here is a clinic case of a 15-year-old patient who underwent diagnosis of dual valve thrombosis who is subsequently taken to fibrinolysis and taking streptokinase without complication and subsequent management with anticoagulation ambulatory therapy.
\end{abstract}

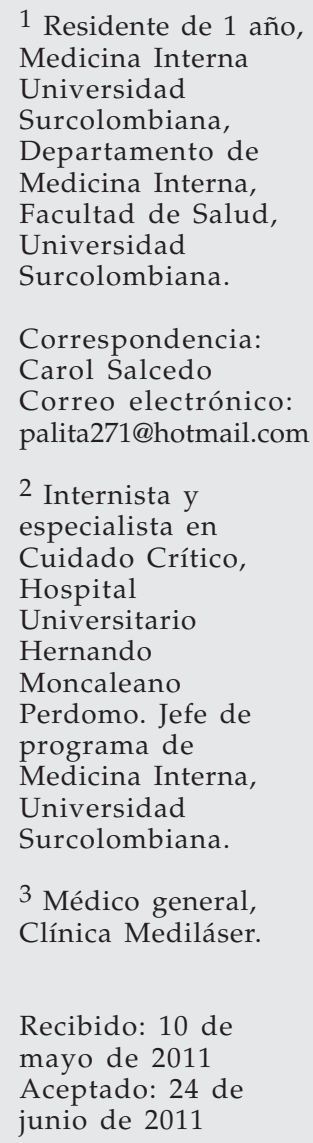

1 Residente de 1 año, Medicina Interna Universidad Surcolombiana, Departamento de Medicina Interna, Facultad de Salud, Universidad Surcolombiana.

Correspondencia: Carol Salcedo Correo electrónico: palita271@hotmail.com

2 Internista y especialista en Cuidado Crítico, Hospital Universitario Hernando Moncaleano Perdomo. Jefe de programa de Medicina Interna, Universidad Surcolombiana.

3 Médico general, Clínica Mediláser.

Recibido: 10 de mayo de 2011 Aceptado: 24 de junio de 2011

\section{Introducción}

La trombosis de las prótesis valvulares cardíacas son una enfermedad grave y complicada que se asocia con alta mortalidad y requiere un buen enfoque clínico con una adecuada utilización de las ayudas diagnósticas como la radiografía de tórax y un estudio ecocardiográfico asociado que permita comprobar la existencia de dicha patología para iniciar un tratamiento inmediato, ya sea con la terapia de trombólisis o con cirugía.

\section{Reporte de caso}

Paciente de 15 años con antecedente de reemplazo valvular mitral y aórtico 11 meses antes del ingreso con implantación de 
prótesis mecánica por fiebre reumática, quien venía recibiendo warfarina $2,5 \mathrm{mg}$ vo cada día y metoprolol $25 \mathrm{mg}$ vo cada $12 \mathrm{~h}$ hasta un mes previo a la admisión: consultó por cuadro clínico de 28 horas de evolución consistente en disnea no sibilante asociado a palpitaciones desencadenadas por medianos esfuerzos, posteriormente disnea de pequeños esfuerzos y ortopnea. 10 horas antes del ingreso tos seca y aparición de dolor opresivo retro esternal, aumento en la sensación de palpitaciones en la valoración inicial, tensión arterial 90/50 mmhg FC 89 x min, frecuencia respiratoria $16 \times \mathrm{min} \mathrm{T}: 37^{\circ} \mathrm{C}$, palidez mucocutánea, mucosas secas, ruidos cardíacos rítmicos, con click valvular normal, murmullo vesicular presente sin agregados pulmonares, no roncus, paraclínicos con leucocitosis de 15,300 y neutrófilos de 77,6\% hemoglobina de 16,1, PT 7,8, PTT 35,3 INR 2,02 , radiografía de tórax compatible con edema pulmonar ya que muestra infiltrados parahiliares bilateral en alas de mariposa, líneas B Kerley, manguitos peribronquiales, presencia de anillo valvular (Figura 1), por lo cual se da tratamiento con oxígeno, carvedilol, warfarina, digoxina y se practica ecocardiograma transesofágico (Tabla 1) que mostró remodelación concéntrica del ventrículo izquierdo de grado leve, buena función sistólica biventricular izquierda, prótesis mecánica de doble disco en posición mitral disfuncional con severo aumento de gradientes, imagen que sugiere trombosis de la valva posterior, prótesis mecánica en posición aórtica normofuncionante, hipertensión pulmonar moderada (PSAP $58 \mathrm{mmhg}$ ), insuficiencia tricúspidea moderada, leve dilatación auricular izquierda con función conservada, corazón hiperdinámico con fc $130 \mathrm{lpm}$.

El paciente es valorado por el servicio de cardiología quien indica trombólisis con estreptoquinasa bolo 250.000 UI y continua con $100.000 \mathrm{UI} / \mathrm{H}$ y es trasladado a la Unidad de Cuidados Intensivos donde posterior a terapia trombolítica se hace radiografía de control que es normal (Figura 2) y ecocardiograma transesofágico con buena función sistólica biventricular, prótesis mecánica en posición aórtica y mitral normofuncionantes, insuficiencia tricúspidea

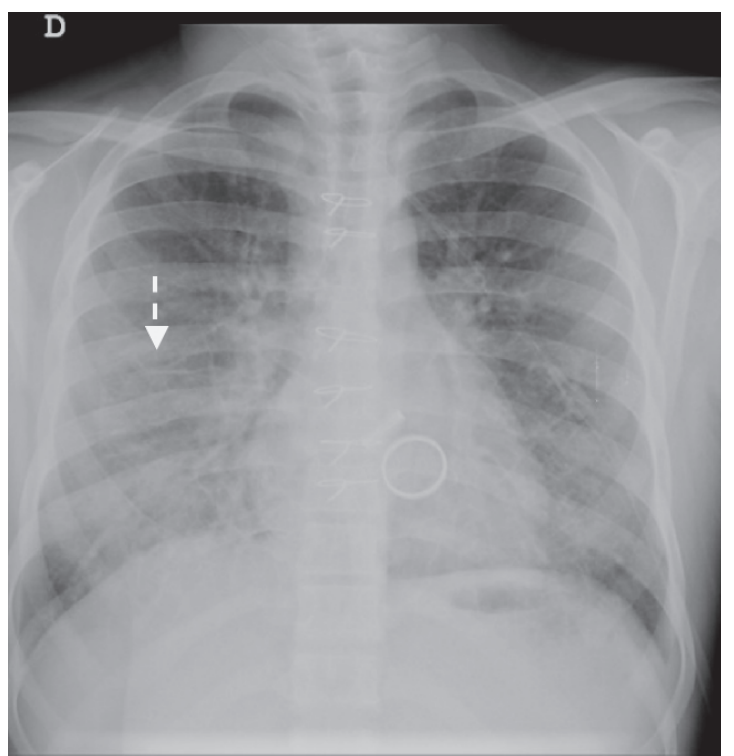

Figura 1. Se muestran infiltrados en alas de mariposa y líneas b Kerley (flecha)

Tabla 1. Ecocardiograma transesofágico $(1 / 3 / 11)$

\begin{tabular}{lc}
\hline F.E. & $\mathbf{6 0} \%$ \\
\hline VI diástole & 4,16 \\
VI sístole & 2,53 \\
Septum & 1,12 \\
Aorta & 1,99 \\
Pared posterior & 0,94 \\
A. izquierda & 4,58 \\
B. derecho & 3,29 \\
Válvula aórtica & G.P 36 \\
PSAP & $58 \mathrm{mmhg}$ \\
\hline
\end{tabular}

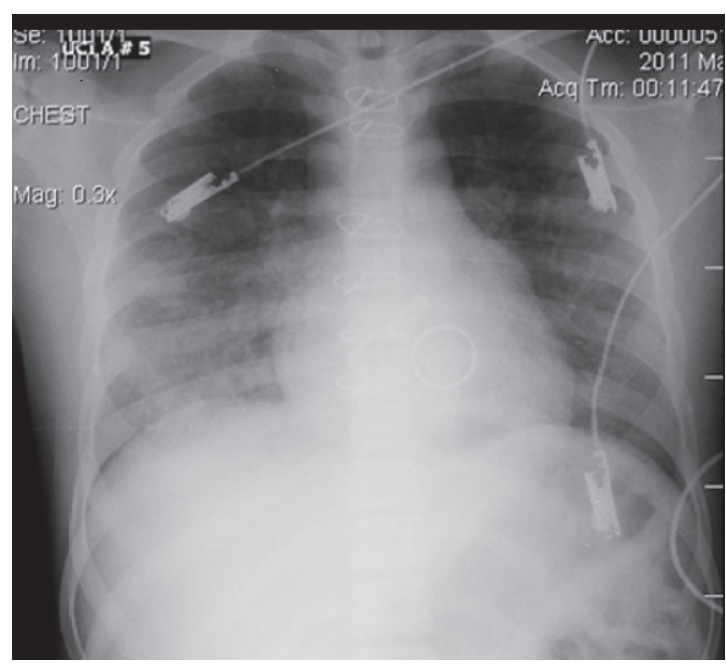

Figura 2. Rx tórax de control (3/4/11) normal 
mínima, estimando una PSAP $37 \mathrm{mmhg}$, hipertensión pulmonar leve, importante mejoría con respecto a estudio previo (Tabla 2), el paciente evoluciona favorablemente por lo que se decide trasladar a piso, iniciar terapia de anticoagulación con warfarina, incentivo respiratorio. Al paciente no se le documenta sangrado, se logra INR 2,98 dándosele salida al octavo día de su ingreso, y se le recomienda continuar controles por consulta externa de cardiología.

Tabla 2. Ecocardiograma transesofágico

\begin{tabular}{lc}
\hline F.E. & $\mathbf{6 0} \%$ \\
\hline VI diástole & 4,50 \\
VI sístole & 3,0 \\
Septum & 0,93 \\
Aorta & 2,16 \\
Pared posterior & 0,86 \\
A. izquierda & 3,25 \\
Válvula mitral & 9,21 \\
Válvula aórtica & G.P 39 \\
PSAP & $37 \mathrm{mmhg}$ \\
\hline
\end{tabular}

\section{Discusión}

Desde 1950 se han utilizado diferentes diseños de prótesis para el reemplazo de válvulas cardíacas; los adelantos en el diseño de las prótesis y avances en la técnica quirúrgica han mejorado considerablemente el pronóstico de los pacientes sometidos a reemplazo valvular(1). Sin embargo la incidencia de trombosis de las prótesis valvulares (TPV) mecánicas puede ser tan alta como del $13 \%$ en el primer año en cualquier posición de la válvula, e incluso del $20 \%$ en posición tricúspidea. Para las prótesis mecánicas en posición mitral y/o aórtica, la incidencia global es de $0,5 \%$ a $6 \%$ por paciente al año, la más alta es en la mitral ${ }^{(2)}$. El riesgo de trombo a pesar de la anticoagulación oral adecuada se ha estimado entre $1 \%$ y $4 \%$ al año ${ }^{(1)}$.

Las válvulas cardiacas enfermas pueden ser reemplazadas por prótesis mecánicas o biológicas $^{(3)}$. Las mecánicas, hechas de metal o diferentes aleaciones de carbono, se clasifican según su diseño en: caja y bola, valva basculante o bivalva. Las biológicas se dividen a su vez en heteroinjertos hechos con tejidos bovinos o porcinos, ya sea del pericardio de la válvula aórtica montada sobre un soporte metálico, o los homoinjertos, que son válvulas humanas aórticas o pulmonares, frescas o preservadas ${ }^{(4)}$.

Las válvulas protésicas mecánicas son más durables, la mayoría duran de 20 a 30 años pero también más trombogénicas que las válvulas biológicas ${ }^{(5)}$. Las ventajas de la bioprótesis sobre las válvulas mecánicas es que proporcionan una hemodinamia más fisiológica y no necesitan a largo plazo de anticoagulación ${ }^{(5)}$. En los pacientes en los cuales se ha practicado cirugías de corrección de válvulas cardiacas con prótesis mecánicas, estas tienden a la formación de trombos, y los factores más importantemente relacionados son la terapia de anticoagulación inadecuada y la localización mitral de la prótesis ${ }^{(2)}$.

Es importante tener en cuenta que existen diferentes tipos de disfunciones protésicas ya sea intrínseca o extrínseca ${ }^{(4)}$, la primera puede ser por un fallo estructural primario de la prótesis o desgaste y rotura del material en las prótesis mecánicas o la degeneración y calcificación de los velos en las prótesis biológicas. En la disfunción protésica extrínseca ocurre por causas ajenas a la propia válvula, como la fuga perivalvular que es la más común y se produce por una separación entre el anillo de la prótesis y el de la válvula sustituida, una técnica quirúrgica deficiente, y requiere una reintervención durante el primer año en la mayoría de los casos. El tamaño inapropiado de la prótesis en relación al anillo receptor (fenómeno llamado "too-small-to fit") en el que la insuficiencia cardiaca alerta sobre esta situación es otra complicación, así como el atrapamiento por pannus, es una complicación tardía, que origina un aumento del gradiente transprotésico con la consiguiente disminución de la capacidad funcional del paciente ${ }^{(4)}$.

La anemia hemolítica por flujo turbulento (fuga, fallo estructural, trombosis, prótesis muy pequeñas) es una complicación menos frecuente, la trombosis protésica que es responsable en el $90 \%$ de las obstrucciones de válvulas protésicas, y la endocarditis protésica 
en la que se afecta a una prótesis valvular o a una válvula nativa reconstruida. Alrededor de un $1-4 \%$ de las prótesis han de sufrir infección a lo largo de la vida del paciente ${ }^{(4)}$.

Los pacientes con trombosis valvular protésica pueden sufrir embolias, edema agudo de pulmón, colapso circulatorio con disnea progresiva y hallazgos típicos que incluyen soplos en la auscultación ${ }^{(6)}$. Los síntomas de presentación de trombosis valvular protésica son mencionados en la Tabla $3^{(7)}$.

Tabla 3. Manifestaciones de la trombosis valvular

\begin{tabular}{ll}
\hline Síntomas y signos & $\%$ \\
\hline La disnea & 56 \\
Ascultación nuevos soplos & 13 \\
Shock & 13 \\
Otros síntomas como dolor abdominal, & \\
síncope o un accidente cerebrovascular & 15 \\
INR 2,5 durante la trombosis & 21 \\
Deterioro de la clase funcional & 87 \\
Desconocidos & 20 \\
\hline
\end{tabular}

El diagnóstico paraclínico debe hacerse inicialmente solicitando cuadro hemático, hemocultivos (en sospechas de endocarditis) gases arteriales, glicemia, electrolitos, tiempos de coagulación en especial tiempo de protrombina e INR para conocer el grado de anticoagulación del paciente, electrocardiograma, además de las imágenes como radiografía de tórax en la que se evalúa signos de hipertensión venocapilar y edema pulmonar, ecocardiograma transtorácico, transesofágico o fluoroscopia ${ }^{(3)}$. En muy pocos casos se requiere de estudio de hemodinamia para confirmar el diagnóstico de disfunción.

El ecocardiograma transtorácico (ETT) y transesofágico (ETE), son los métodos diagnósticos de mayor sensibilidad y especificidad, para confirmar la sospecha clínica de disfunción de la prótesis valvular. Estos métodos pueden demostrar la disminución de movimiento del mecanismo de cierre y determinar el aumento de gradiente sistólico o diastólico transvalvular, comparado con los datos posoperatorios tempranos al egreso de cirugía(3). La fluoroscopia simple o con amplificación de imagen se utiliza para observar la limitación del movimiento de la bola o de las valvas de las prótesis mecánicas en caso de trombosis, para analizar el cabeceo de la prótesis ${ }^{(3)}$. En el paciente inestable lo ideal es realizar Ecocardiograma transtorácico y el paciente estable requiere fluoroscopia, ecocardiograma transtorácico y transesofagico ${ }^{(4)}$.

En el ecocardiograma se debe inicialmente valorar el tamaño del coágulo y con esto se puede definir conducta de anticoagulación versus cirugía o trombólisis ${ }^{(5)}$ (Figura 3).

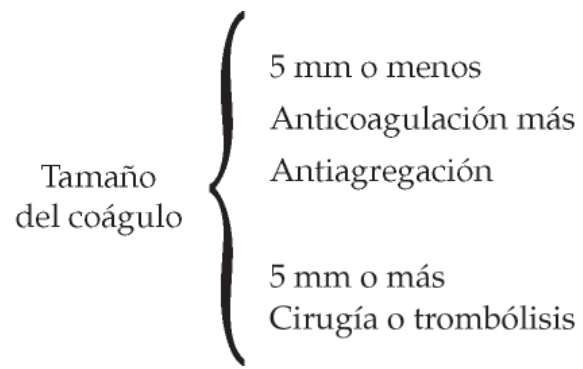

Figura 3. Conducta a seguir de acuerdo al tamaño del coágulo

\section{Características de la terapia}

La TVP es una complicación seria asociado con una alta mortalidad y requiere diagnóstico y tratamiento inmediato ${ }^{(3)}$. El manejo óptimo de estos pacientes es motivo de controversia en la actualidad; la trombectomía o el reemplazo valvular son los tratamientos de elección ${ }^{(8)}$.

La terapia quirúrgica tiene una mortalidad del $15 \%$, esta se incrementa en cirugía de urgencia e inestabilidad hemodinámica pero tiene menor recurrencia ${ }^{(9)}$. Por su parte la trombólisis tiene una mortalidad de 9$11 \%$, con un éxito del $70 \%$, que aumenta en pacientes con síntomas de $<$ de 2 semanas, el riesgo de embolismo es del $19 \%{ }^{(9)}$.

Los trombolíticos que se usan actualmente son rtPA $10 \mathrm{mg}$ en forma de bolo intravenoso, seguido por una infusión de 90 mg durante 90 min; estreptoquinasa 250500.000 UI durante 20 min seguido por $1 \mathrm{a}$ 1,5 millones de UI en infusión durante $10 \mathrm{~h}$. Después de la trombólisis se debe administrar heparina no fraccionada por infusión intravenosa en todos los pacientes (tiempo parcial de activación de tromboplastina 1,5- 
2,0 veces el control). La urocinasa también es una alternativa, en una dosis de $4.400 \mathrm{U} /$ $\mathrm{kg} / \mathrm{h}$ sin heparina durante $12 \mathrm{~h}, \mathrm{o} 2.000 \mathrm{U} /$ $\mathrm{kg} / \mathrm{h}$ con heparina durante $24 \mathrm{~h}$ (nueva) ${ }^{(10)}$, en el caso clínico se usó estreptoquinasa y no rtPa porque es la más accesible en nuestra institución, además de los bajos costos que tiene. Se han empleado múltiples formas de terapia trombolítica, aunque la mayor experiencia es con la estreptoquinasa(11).

La urocinasa se ha utilizado de manera eficaz y el activador hístico del plasminógeno tiene la ventaja de alcanzar su efecto farmacológico máximo en menor tiempo y se revierte con más rapidez. En un estudio en donde se empleo un protocolo de tratamiento en 25 pacientes en donde se administró estreptoquinasa (SKr) por vía intravenosa periférica, a una dosis inicial de 250000 UI en 30 minutos, seguida de una infusión a 100000 UI/ por 72 horas (o menos tiempo) si existió evidencia clínica y/o ecocardiográfica de resolución de la trombosis protésica valvular logrando un buen nivel de éxito y bajo índice de embolismo con las dosis lentas. El tratamiento fue exitoso en 24 pacientes (96\%), con respuesta total en $21(84 \%)$, parcial en $3(12 \%)$ y fracasó en 1 (quien falleció durante la intervención quirúrgica posterior a la trombólisis), además se observaron complicaciones en 5 pacientes $(20 \%)$, con embolismo cerebral en un paciente $(4 \%)$, con ataque transitorio isquémico y hemorragia intracraneal en otro (4\%). Ambos pacientes fueron tratados médicamente con resolución de las manifestaciones cerebrovasculares y mejoría clínica, y ecocardiográfica, sin secuelas al alta hospitalaria(11).

Las recomendaciones de la Sociedad Europea de Cardiología es practicar manejo quirúrgico excepto en los siguientes $\operatorname{casos}^{(12)}$ :

- Críticamente enfermos (inestables).

- Corazón derecho (menor riesgo de embolismo).

- No disponibilidad de cirugía.

Un estudio recientemente publicado en el que se comparó las dos opciones de tratamiento; en el primero se practicó trombólisis con rt-PA a dosis de $10 \mathrm{mg}$ en bolo y $90 \mathrm{mg}$ en 90 min a un grupo de 13 pacientes, se evidenció $92 \%$ de mejoría pero dentro de las complicaciones que se presentaron se informó: un accidente isquémico transitorio, un evento cerebrovascular (ECV), dos embolias periféricas con recuperación total y una complicación que requirió cirugía ${ }^{(9)}$; en el grupo operatorio se estudiaron 18 pacientes con 2 muertes (11\%) en fase perioperatoria, 2 recurrencias de TVP durante una mediana de seguimiento de 76 meses y otras complicaciones esternitis y sepsis con estancias prolongadas en la Unidad de Cuidados Intensivos de más de 4 semanas en 2 pacientes. Se llegó a la conclusión de que las complicaciones del rt-Pa son transitorias y de naturaleza benigna, el ECV que es la más seria complicación, fue hallada en 1 paciente, en cambio la mortalidad quirúrgica fue de 2 pacientes y se presentó aumento en el tiempo de hospitalización hasta 8 semanas en 2 pacientes ${ }^{(9)}$.

Hay que tener en cuenta que la reparación y la sustitución de la válvula mitral son dos técnicas terapéuticas independientes para el tratamiento de la enfermedad de la válvula mitral. La comunidad quirúrgica se ha vuelto más consciente de las ventajas conocidas del reemplazo frente a la reparación de la valvular mitral y por lo cual diferentes estudios asocian una menor tasa de tromboembolismo y de infección a la sustitución de la válvula(12).

Cuando existe una trombosis de la válvula protésica del lado izquierdo del corazón, la cirugía es usualmente el tratamiento de elección, particularmente en casos de obstrucción crónica u obstrucción postoperatoria temprana, sin embargo se propone la realización de fibrinólisis en pacientes críticamente enfermos (edema pulmonar, hipotensión, clase funcional III/IV) con obstrucción aguda si no es posible la realización de cirugía inmediata, o existe alguna contraindicación para el procedimiento (bajo gasto cardiaco, insuficiencia respiratoria, reintervención) $^{(13)}$ (ver Figura 4).

Aunque no existen estudios con muestras grandes que determinen la efectividad de las terapias para TVP, una variedad de recomendaciones propuestas por expertos son expuestas en la literatura y en este artículo. 


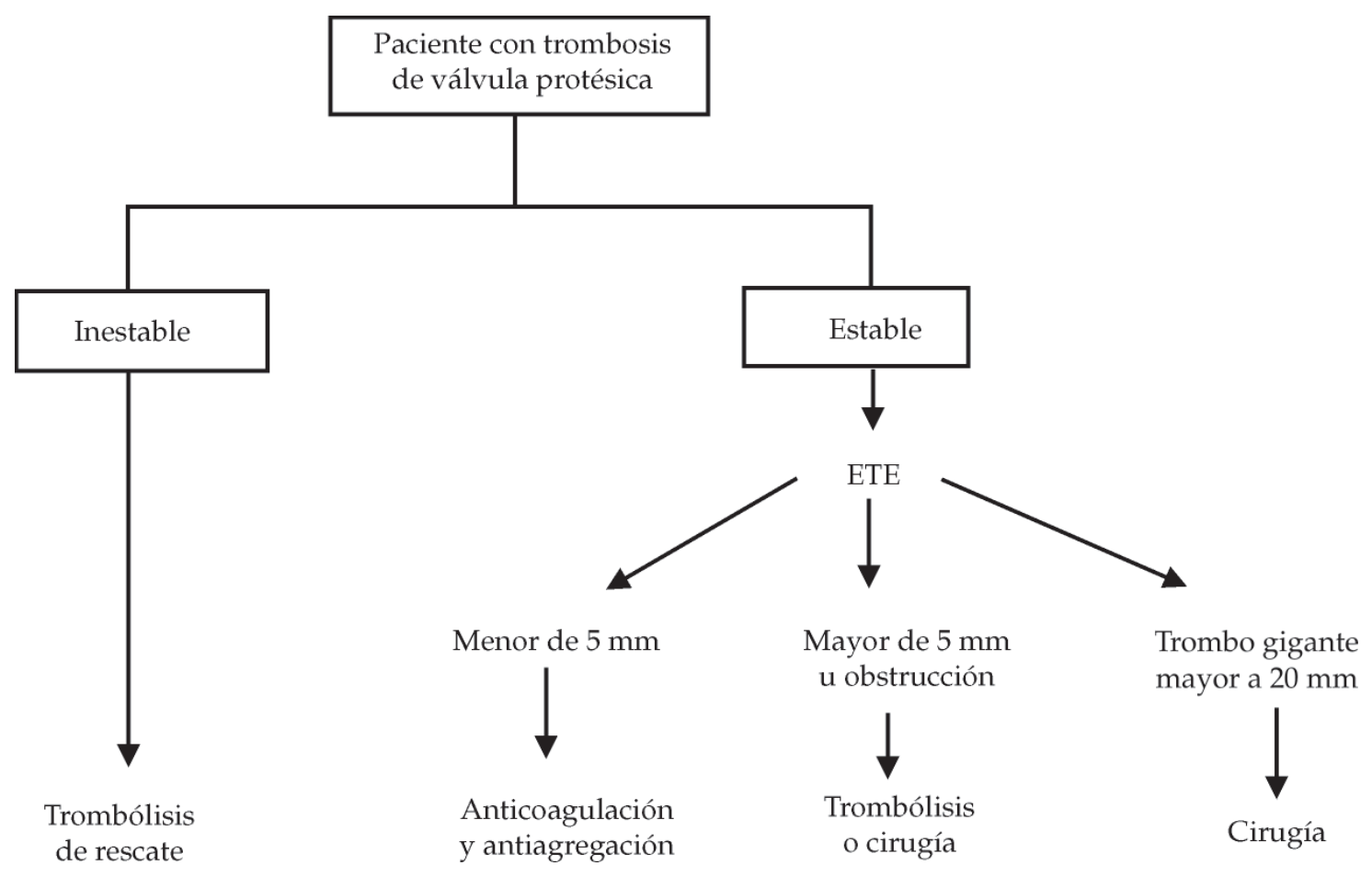

Figura 4. Algoritmo de manejo de TVP

\section{Referencias Bibliográficas}

1. Cáceres F, Pérez H. 2006. Prosthetic heart valve thrombosis: Pathogenesis, diagnosis and management. International Journal of Cardiology 110:1-6.

2. Maribas P. 2009. Management of prosthetic heart valve obstruction: Speech for the surgery?. Archives of Cardiovascular Disease. 102: 255-257.

3. Uribe C. 2006. Disfunción de prótesis valvulares. Manual de Urgencia cardiovascular, fundación Abood Shaio 102:221-227.

4. Bonow R, Carabello B. 2008. ACC/AHA guidelines for the management of patients with valvular heart disease: a report of the American College of Cardiology/American Heart Association Task Force on practice guidelines (committee on management of patients with valvular heart disease). J Am Coll Cardiol 52:e104-e110.

5. Sun J, Davidson M. 2009. Antithrombotic management of patients with prosthetic heart valves: current evidence and future trends. Lancet 374:565-576.

6. Gündüz S, Özkan M. 2009. Mechanical mitral valve thrombosis and giant left atrial thrombus: comparison of transesophageal echocardiography and 64-slice multidetector computed tomography. Arch Turk Soc Cardiol 37(7):483-487.
7. Durrleman N, Pellerin M. 2004. Prosthetic valve thrombosis: twenty-year experience at the Montreal Heart Institute. J Thorac Cardiovasc Surg 127:1388-1392.

8. Roudaut R, Lafitte S. 2003. Fibrinolysis of Mechanical Prosthetic Valve Thrombosis A Single-Center Study of 127 Cases. Journal of the American College of Cardiology 41:653-658.

9. Keuleers S, Herijgers P. 2011. Comparison of thrombolysis versus surgery as a first line therapy for prosthetic heart valve the thrombosis. American Journal of Cardiology 107:275-279.

10. Nieminen MS. et al. 2005. Guías de la Sociedad Europea de Cardiología: insuficiencia cardíaca aguda 58(4):389-429. P. 415-416.

11. Cáceres Lóriga F, Pérez López H. 2005. Tratamiento trombolítico en la trombosis valvular protésica. Experiencia en el Instituto de Cardiología de Cuba. Mapfre Medicina 16:8185.

12. Roudaut R, Lafitte S. 2009. Management of prosthetic heart valve obstruction: Fibrinolysis versus surgery. Early results and long-term follow-up in a single-centre study of 263 cases. Archives of Cardiovascular Disease 102:269-277.

13. Shuhaiber J, Anderson R. 2007. Meta-analysis of clinical outcomes following surgical mitral valve repair or replacement. European Journal of Cardio-thoracic Surgery 31:267-275. 